\title{
Achieving and Maintaining Cardiovascular Health Across the Lifespan
}

\author{
Kunal N. Karmali • Donald M. Lloyd-Jones
}

Published online: 2 April 2014

(C) Springer International Publishing AG 2014

\begin{abstract}
The American Heart Association introduced the concept of cardiovascular health $(\mathrm{CVH})$ in its 2020 Strategic lmpact Goals. Defined by the presence of four health behaviors (smoking, weight, diet, and physical activity) and three health factors (glucose, blood pressure, and cholesterol), $\mathrm{CVH}$ reflects an expansion of preventive efforts to the entire population and a reframing of disease prevention to health promotion. Recent evidence has confirmed the relevance of the seven CVH metrics in cardiovascular outcomes, highlighting the critical role of a healthy lifestyle in achieving and maintaining $\mathrm{CVH}$ through the lifespan. Primordial prevention efforts geared towards health promotion and healthy behaviors, sustained over the life course, and fostered by public health and health policy will be the key to achieving and maintaining $\mathrm{CVH}$ and improving the cardiovascular health of the nation.
\end{abstract}

Keywords Cardiovascular health $\cdot$ Lifestyle $\cdot$ Primordial prevention $\cdot$ Cardiovascular disease $\cdot$ Risk factors

\section{Introduction}

In 2010, the American Heart Association (AHA) announced a new focus for its 2020 Strategic Impact Goals: "By 2020, to improve the cardiovascular health of all Americans by $20 \%$ while reducing deaths from cardiovascular diseases and stroke by $20 \%$." [1••] These goals reflected an expansion of

\section{K. N. Karmali • D. M. Lloyd-Jones $(\bowtie)$}

Department of Medicine-Cardiology and Department of Preventive Medicine, Northwestern University Feinberg School of Medicine, 680 North Lake Shore Drive, Suite 1400, Chicago, IL 60611, USA

e-mail: dlj@northwestern.edu

K. N. Karmali

e-mail: kunal-karmali@northwestern.edu prevention efforts to the entire population and introduced the concept of cardiovascular health $(\mathrm{CVH})$, a positive construct conceptualizing health and prevention as more than the absence of clinically evident disease. The AHA developed four health behavior metrics (smoking status, body mass index, dietary content, and participation in physical activity) and three health factor metrics (blood glucose, blood pressure, and total cholesterol) that, taken together, define CVH. Within each health metric, three clinically based strata of poor, intermediate, and ideal were set to capture the full spectrum of $\mathrm{CVH}$. Since the AHA announced its 2020 Strategic Impact Goals, multiple studies have confirmed the importance of the $\mathrm{CVH}$ construct and its relevance to both cardiovascular and overall health outcomes. In this review, we describe the conceptual framework of $\mathrm{CVH}$, highlight recent data demonstrating its relevance to health outcomes, and provide key strategies needed to achieve and maintain $\mathrm{CVH}$ over the lifespan.

\section{Cardiovascular Health}

The shift in focus from cardiovascular disease prevention to cardiovascular health promotion has been called a public health revolution [2]. Although origins can be traced back to the World Health Organization Constitution of 1946 and the Surgeon General's Healthy People report in 1979, CVH as a construct defined by measureable health behavior and health factor metrics was first established by the AHA 2020 Strategic Impact Goals $[1 \bullet \cdot, 2]$. The goal was to reframe preventive efforts broadly to promote health and health behaviors in the population for greater societal and public health impact. Three central ideas underscore this new framework: 1) the balance between population- and individual-level interventions, 2) the significance of primordial prevention, and 3) the need to promote and maintain healthy behaviors and minimize risk factors throughout the lifespan. 
The first concept was popularized by Geoffrey Rose, who described the complementary relationship of preventive efforts directed towards unhealthy individuals (the high-risk strategy) and unhealthy populations (the population-based strategy) [3]. Because the majority of cardiovascular disease (CVD) events occur in the large segment of the population with average or only mildly elevated risk factors, Rose argued that population-level strategies could more effectively prevent CVD events by shifting the entire distribution of risk [3]. Small changes in mean population levels of risk would then result in large reductions in disease rates. The idea of "primordial prevention" was first introduced by Toma Strasser in 1978 [4]. This concept acknowledges that adverse risk factors carry graded and continuous cardiovascular risk and that pharmacologic and lifestyle interventions for secondary and primary prevention will not reduce event rates to rates seen in people who maintain optimal risk factor profiles throughout the lifespan. Therefore, prevention of risk factor development in the first place can hold immense societal and public health impact. The $\mathrm{CVH}$ construct attempts to integrate these two philosophies and incorporate them over the lifespan to reframe CVD prevention as lifelong health promotion.

The centerpiece of the AHA 2020 Strategic Impact Goals is ideal CVH, defined as the absence of clinically manifest CVD together with the simultaneous presence of optimal levels of all seven health metrics (four health behaviors and three health factors) $[1 \bullet \bullet$. However, the CVH construct also recognizes that cardiovascular health can occur across a spectrum and therefore clinically based strata of "poor," "intermediate," and "ideal" levels for each health metric were defined (Table 1). For individuals with a history of clinical CVD, ideal CVH is not achievable, but controlling all health factors and health behaviors can move an individual from poor $\mathrm{CVH}$ to intermediate $\mathrm{CVH}$. Among individuals without clinical CVD who have poor $\mathrm{CVH}$, controlling all health factors and health behaviors with lifestyle or medications can help move one from poor to intermediate levels of $\mathrm{CVH}$ or from intermediate to ideal $\mathrm{CVH}$ if such control is achieved with lifestyle alone $[1 \bullet \bullet]$.

\section{Relevance of Cardiovascular Health}

Although evidence had previously assessed clusters of ideal levels of risk factors with longevity [5], disease-free survival [6], quality of life [7], and healthcare costs [8, 9], only in the past few years have empirical data emerged to support the $\mathrm{CVH}$ construct. Analyses from the Atherosclerosis Risk in Communities study first demonstrated the link between $\mathrm{CVH}$ and outcomes. In the study, Folsom et al. analyzed 12,744 participants without CVD and demonstrated a step-wise inverse association between the number of ideal health metrics and incident CVD events (including coronary heart disease death, nonfatal myocardial infarction, stroke, and heart failure) during 20 years of follow-up. Individuals with six or more ideal health metrics had approximately one tenth the rate of 20-year incident CVD compared with individuals with zero ideal health metrics, 3.9 events per 1,000 person-years vs. 32.1 events per 1,000 person-years (hazard ratio (HR) 0.11 , $95 \%$ confidence interval (CI) $0.07-0.17$ ) [10]. In the 17 middle-aged participants with all seven metrics rated ideal, no CVD events were seen in the 20 years of follow-up. In contrast, CVD incidence rates were 7.5 per 1,000 person-years (95\% CI 6.4-8.4) for those with intermediate CVH and 14.6 per 1,000 person-years (95\% CI 14.0-15.2) for those with poor CVH. Similar analyses from the Northern Manhattan Study including a multi-ethnic cohort of whites, blacks, and Caribbean Hispanics from the New York area demonstrated an analogous dose response relationship between the number of ideal $\mathrm{CVH}$ metrics and individual CVD endpoints, confirming the relevance of $\mathrm{CVH}$ regardless of race-ethnic background.

Nationally representative data from the National Health and Nutrition Examination Surveys (NHANES) have also shown similar relationships. Using NHANES follow-up data from 1988 to 2006, Yang et al. reported a stepwise association between the number of ideal CVH metrics and the risk of allcause mortality, CVD mortality, and ischemic heart disease mortality [11•]. In those who met six or more metrics, the adjusted HRs compared with individuals with one or fewer ideal health metrics was 0.49 (95\% CI 0.33-0.74) for allcause mortality, 0.24 (95\% CI 0.13-0.47) for CVD mortality, and 0.30 (95\% CI 0.13-0.68) for ischemic heart disease mortality. Short-term data from more recent NHANES surveys (1999-2006) have demonstrated similar relationships [12].

The benefits of CVH metrics have also been linked to noncardiovascular conditions. For example, Rasmussen-Torvik et al. demonstrated a graded inverse association between adherence to the AHA's seven CVH metrics and cancer incidence over a 19-year follow-up with a $51 \%$ lower risk of cancer among individuals with six or seven ideal CVH metrics compared with those with zero ideal metrics (HR 0.49, $95 \%$ CI 0.35-0.69) [13]. This relationship remained significant even after excluding smoking status from the ideal health score. Similar prospective benefits in important noncardiovascular outcomes have also been demonstrated for cognition [14], depressive symptoms [15], and health-related quality of life [16]. These data solidify the case that better $\mathrm{CVH}$ is associated with overall healthier longevity. Recent data support this notion by demonstrating greater long-term disease-free longevity and compression of morbidity in middle-aged men and women with better CVH [6]. 


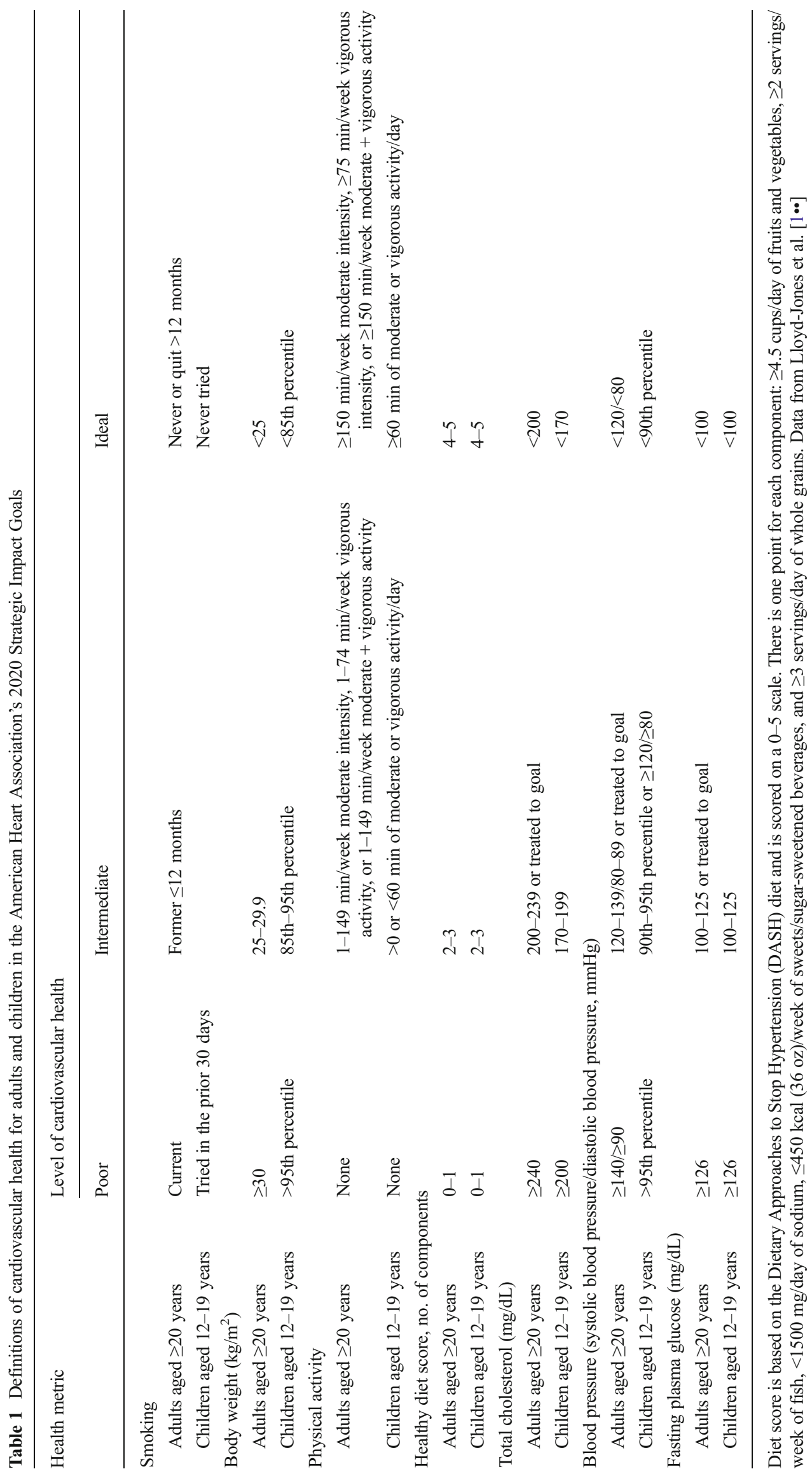




\section{Prevalence of Cardiovascular Health in the USA}

Although studies have convincingly demonstrated the health benefits of the ideal $\mathrm{CVH}$ construct, prevalence of ideal $\mathrm{CVH}$ metrics has consistently been low in community samples [17], longitudinal cohort studies [10, 18], and nationally representative surveys $[11 \cdot, 12,19]$. Longitudinal analyses of multiple NHANES surveys demonstrated prevalence of ideal CVH of less than $2 \%$ that remained similarly low over time [11•]. The most up-to-date survey data from the NHANES 20092010 cycle estimates that only $0.1 \%$ of US adults aged $\geq 20$ years meet all seven ideal CVH criteria [20]. Moreover, state-by-state assessments of $\mathrm{CVH}$ metrics reveal significant geographic variation, with prevalence of ideal $\mathrm{CVH}$ from $1.2 \%$ in Oklahoma to $6.9 \%$ in the District of Columbia, and poor $\mathrm{CVH}$ from $6.7 \%$ in Colorado to $16.2 \%$ in West Virginia [21].

These sobering prevalence estimates of $\mathrm{CVH}$ underscore the pervasiveness of unhealthy behaviors and risk factors and the enormous challenge we face to achieve $\mathrm{CVH}$ in the population. However, these same prevalence data hint at a pathway toward greater $\mathrm{CVH}$ in the population. The presence of ideal CVH is age related with younger adults more likely to meet greater numbers of ideal metrics than older adults (Fig. 1). For example, nearly half of US children (aged 1219 years) meet five or more CVH criteria whereas only $17 \%$ of US adults aged $\geq 20$ years meet five or more criteria [20]. Thus, we are born with a stock of ideal CVH and this health is lost during adolescence and young adulthood through the adoption of unhealthy behaviors related to poor diet, sedentary lifestyle, obesity, and smoking. Achievement of ideal CVH into adulthood may thus be best reached by maintaining it over the lifespan, rather than attempting to restore it. With the inclusion of health behaviors in the definition of $\mathrm{CVH}$, the primacy of lifestyle and health behaviors as the antecedents of risk factors is firmly acknowledged. Moreover, the critical role of societal factors as an enabler of unhealthy behaviors and a barrier to healthy ones is also emphasized, thus justifying the role for public health and policy initiatives geared toward promoting health.

\section{Adopting a Life-Course Approach to Cardiovascular Health}

From early pathologic studies such as those in Korean War casualties [22] to studies in childhood cohorts [23, 24], there has been increasing evidence that atherosclerosis originates in childhood and is influenced by early risk factor levels and unhealthy behaviors. Pooled data from four prospective cohorts, the Bogalusa Heart Study, Cardiovascular Risk in Young Finns Study, Childhood Determinants of Adult Health study, and the Muscatine Study, have tracked these lifestyle and biologic factors into adulthood to demonstrate that higher risk-factor levels in youth are significantly associated with carotid intima-media thickness (IMT) in adulthood [25]. Similar analyses from the Coronary Artery Disease in Young Adults (CARDIA) cohort have shown an analogous relationship between young adulthood risk factor levels and coronary artery calcification in middle age [26].

These cohort analyses have been extended to demonstrate the importance of childhood achievement of ideal CVH on intermediate outcomes in adulthood. Using data from the Young Finns Study, Laitinen et al. examined the effects of the seven CVH metrics on cardiometabolic outcomes in adulthood in 856 Finnish participants aged 12-18 years [27••]. With up to 21 years of follow-up, each unit increase in ideal CVH metric in childhood was associated with a lower risk of hypertension (odds ratio (OR) 0.66, $95 \%$ CI 0.54-0.80), metabolic syndrome (OR 0.63, $95 \%$ CI 0.52-0.77), elevated low-density lipoprotein cholesterol (OR 0.66, $95 \%$ CI 0.52 0.85 ), and high-risk IMT (OR 0.75, $95 \%$ CI 0.60-0.94) in adulthood. Ideal childhood $\mathrm{CVH}$ was inversely associated
Fig. 1 Prevalence estimates of US individuals meeting different numbers of criteria for ideal cardiovascular health by age groups, National Health and Nutrition Examination Surveys 2009-2010. Prevalence of ideal levels of cardiovascular health metrics are higher in US children (aged 12-19 years) and young adults (aged $20-39$ years) than in older adults. Data from Go et al. [20]
Prevalence of CVH in NHANES 2009-2010 by age group

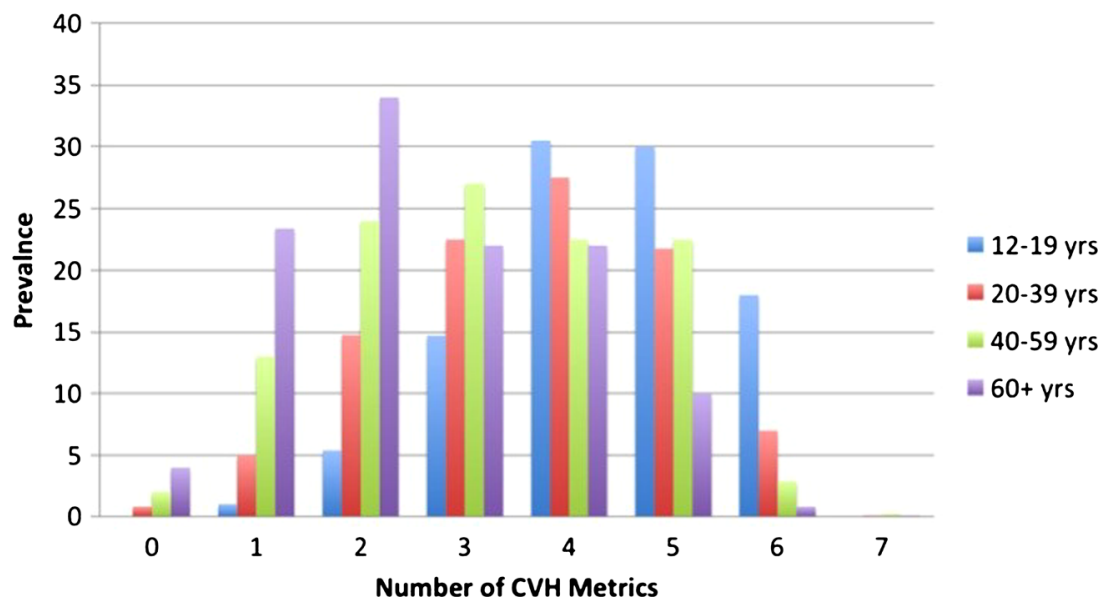


with adult carotid IMT with a $0.067-\mathrm{mm}$ difference in adult carotid IMT between children with six criteria for CVH compared with one criterion. To put this in clinical context, a 0.1$\mathrm{mm}$ increase in carotid IMT has been demonstrated to be predictive for myocardial infarction (HR 1.15, $95 \%$ CI 1.12-1.18) and for stroke (HR 1.17, $95 \%$ CI 1.15-1.21) [28]. Thus, these findings emphasize the role of childhood $\mathrm{CVH}$ for future outcomes in adults and highlight the importance of a life-course approach to $\mathrm{CVH}$.

\section{The Importance of Health Behaviors}

Analyses from CARDIA and the Framingham Heart Study have provided key insights into the role of health behaviors in maintaining $\mathrm{CVH}$ from young adulthood to middle age. In an analysis of 3,154 black and white participants aged 1830 years from the CARDIA study, Liu et al. tested the relationship between adopting healthy lifestyle factors during young adulthood and prevalence of low CVD risk in middle age $[29 \bullet \bullet]$. Healthy lifestyle behaviors consisted of the four health behaviors in the CVH metric (body mass index $<$ $25 \mathrm{~kg} / \mathrm{m}^{2}$, healthy diet, physical activity, nonsmoking) with the addition of no or moderate alcohol intake. Participants had data collected at year 0 (baseline), year 7 , and year 20 . Among those who practiced all five healthy lifestyle behaviors in young adulthood, approximately $60 \%$ had low CVD risk in middle age (Table 2). Furthermore, there was a dose-response relationship between the number of healthy lifestyle behaviors followed and the prevalence of low CVD risk 20 years later. Although individuals who maintained a healthy lifestyle throughout the 20 years had the lowest risk for CVD, there was still an important role for behavioral change. Among

Table 2 Low-risk status at year 20 by number of healthy lifestyle factors, year 0 to year 20, among Coronary Artery Disease in Young Adults (CARDIA) study participants $(n=3,154)$

\begin{tabular}{ll} 
Healthy lifestyle factors & $\begin{array}{l}\text { Prevalence of low cardiovascular } \\
\text { risk profile (\%) }\end{array}$ \\
\hline $0-1$ & 3.0 \\
2 & 14.6 \\
3 & 29.5 \\
4 & 39.2 \\
5 & 60.7
\end{tabular}

Healthy lifestyle factors include: body mass index $<25 \mathrm{~kg} / \mathrm{m}^{2}$, average alcohol intake of $0-15 \mathrm{~g} / \mathrm{day}$ for women and $0-30 \mathrm{~g} /$ day for men; highest $40 \%$ on a dietary score; highest $40 \%$ of the sex-specific distribution of average physical activity; and never smoking. Estimates were based on the average values from the baseline exam, year 7, and year 20. Low cardiovascular risk profile was defined as the absence of pre-existing cardiovascular disease and the simultaneous presence of untreated cholesterol $<200 \mathrm{mg} / \mathrm{dL}$, untreated blood pressure $<120 /<80 \mathrm{mmHg}$, no diabetes mellitus, and never smoking. Data from Liu et al. [29••] those who had four or fewer healthy lifestyle behaviors at all time points, the prevalence of low CVD risk at year 20 was only $13.6 \%$. Conversely, for those who had four or fewer healthy lifestyle behaviors at baseline but then had four or more healthy behaviors at year 7 , year 20 , or both, the prevalence of low CVD risk was higher $(29.8 \%, 37.5 \%$, and $52.2 \%$, respectively). These results indicate that regardless of timing, adoption of healthy lifestyle has benefit for the primordial prevention of CVD risk factors and the preservation of $\mathrm{CVH}$.

Analyses of the Framingham Heart Study provide further support to the critical role of health behaviors and lifestyle factors in achieving $\mathrm{CVH}$. Among related members of the Original, Offspring, and Third Generation of the Framingham Heart Study cohorts who had risk factors measured at similar ages, the heritability of ideal CVH was modest, varying from 13 to $18 \%$. Similar analyses in a twin sample showed that the inverse association between CVH metrics and carotid IMT was independent of shared genetic and familial factors [30]. Thus, the majority of $\mathrm{CVH}$ was explained by the environment related to health behaviors and lifestyle factors rather than heritability and genetics [31].

The health behaviors that make up the $\mathrm{CVH}$ construct provide specific targets for behavior change. A recent AHA Scientific Statement identified several, effective, clinical interventions for achieving behavior change including goal setting, motivational interviewing, regular feedback, increasing self-efficacy, and multicomponent behavioral interventions [32]. Mobile technologies have also been used to facilitate weight loss and multi-behavioral interventions [33, 34•]. More research is still needed to examine which approaches lead to meaningful, long-term, behavior change that will impact clinical outcomes such as CVD, mortality, hospitalizations, and quality of life.

\section{Public Policy}

Although personal responsibility and individual-level clinical interventions are essential, population-based interventions will also be critical in achieving greater $\mathrm{CVH}$ in the nation. This population-based strategy is rooted in the social ecological theory that sees an individual's social and physical environment as a strong potential influence on health and its determinants [35]. The loss of CVH therefore reflects systemic deficiencies in the environment that: facilitate sedentary behaviors; promote consumption of high-calorie nutrientpoor foods; and impede healthy weight maintenance. As proposed by Geoffrey Rose, population-based strategies are essential to shift the population towards healthier behaviors [3]. Successful public health interventions promoting $\mathrm{CVH}$ include sodium reduction in processed foods, public smoking bans and tobacco excise taxes to limit smoking, and 
modifications of the built environment to promote physical activity. These strategies help maintain optimal levels of CVH and shift the entire distribution of risk to lower levels. A recent AHA Scientific Statement systematically reviewed evidence for various population strategies across multiple domains including media, product labeling, taxation, school and workplace initiatives, local environment changes, and legal mandates [36]. New laws such as the passage of the 2010 Patient Protection and Affordable Care Act has provided the health community with multiple opportunities to improve access to clinical preventive services, bolster workplace wellness, and strengthen the role of communities in promoting prevention [37]. Moreover, the Department of Health and Human Services has partnered with state and local governments to launch the "Million Hearts" initiative to coordinate and enhance CVD prevention programs across all government health agencies to prevent 1 million heart attacks and strokes over the next 5 years [38]. Such policy initiatives attempt to enhance and restructure the environment where people live, making healthy behaviors and healthy choices the norm.

\section{Conclusions}

The AHA's 2020 Strategic Impact Goals set out a bold new strategy to improve the cardiovascular health of the nation. The CVH construct was defined and quantified based on seven health behaviors and factors. A combination of clinical, public health, and health policy interventions will be critical to reduce the prevalence of poor $\mathrm{CVH}$, and increase the control of risk factors and improve the prevalence of ideal and intermediate levels of CVH. For each metric, modest shifts in the population distribution towards improved health can produce large increases in the proportion of Americans in the idealand intermediate-health categories, producing substantial cardiovascular benefits. The strong correlations between health behaviors and ideal health factors suggest that individuals can achieve and maintain cardiovascular health through health behaviors rather than through genetic predisposition. Primordial prevention efforts geared toward health promotion, sustained over the life course, and fostered by public health and health policy will be the key to preserving and maintaining $\mathrm{CVH}$ from childhood throughout the lifespan and improve the cardiovascular health of the nation.

\section{Compliance with Ethics Guidelines}

Conflict of Interest KN Karmali declares no conflicts of interest. DM Lloyd-Jones declares no conflicts of interest.

Human and Animal Rights and Informed Consent This article does not contain any studies with human or animal subjects performed by any of the authors.

\section{References}

Papers of particular interest, published recently, have been highlighted as:

- Of importance

•- Of major importance

1.• Lloyd-Jones DM, Hong Y, Labarthe D, Mozaffarian D, Appel LJ, Van Horn L, et al. Defining and setting national goals for cardiovascular health promotion and disease reduction: the American Heart Association's Strategic Impact Goal through 2020 and beyond. Circulation. 2010;121(4):586-613. Introduces the new "cardiovascular health" construct adopted by the American Heart Association for its 2020 Strategic Impact Goal. Ideal health behaviors and factors are: nonsmoking, body mass index $<25 \mathrm{~kg} / \mathrm{m}^{2}$, physical activity, healthy diet, untreated cholesterol $<200 \mathrm{mg} / \mathrm{dL}$, untreated blood pressure $<120 / 80 \mathrm{mmHg}$, and fasting blood glucose $<100 \mathrm{mg} / \mathrm{dL}$.

2. Labarthe DR. From cardiovascular disease to cardiovascular health: a quiet revolution? Circ Cardiovasc Qual Outcomes. 2012;5(6): e86-92.

3. Rose G. Sick individuals and sick populations. Int J Epidemiol. 1985;14(1):32-8.

4. Strasser T. Reflections on cardiovascular diseases. Interdiscip Sci Rev. 1978;3(3):225-30.

5. Stamler J, Stamler R, Neaton JD, Wentworth D, Daviglus ML, Garside D, et al. Low risk-factor profile and long-term cardiovascular and noncardiovascular mortality and life expectancy: findings for 5 large cohorts of young adult and middle-aged men and women. JAMA. 1999;282(21):20128.

6. Wilkins JT, Ning H, Berry J, Zhao L, Dyer AR, Lloyd-Jones DM. Lifetime risk and years lived free of total cardiovascular disease. JAMA. 2012;308(17):1795-801.

7. Daviglus ML, Liu K, Pirzada A, Yan LL, Garside DB, Feinglass J, et al. Favorable cardiovascular risk profile in middle age and healthrelated quality of life in older age. Arch Intern Med. 2003;163(20): 2460-8.

8. Daviglus ML, Liu K, Greenland P, Dyer AR, Garside DB, Manheim L, et al. Benefit of a favorable cardiovascular riskfactor profile in middle age with respect to Medicare costs. N Engl J Med. 1998;339(16):1122-9.

9. Daviglus ML, Liu K, Pirzada A, Yan LL, Garside DB, Greenland $\mathrm{P}$, et al. Cardiovascular risk profile earlier in life and Medicare costs in the last year of life. Arch Intern Med. 2005;165(9):1028-34.

10. Folsom AR, Yatsuya H, Nettleton JA, Lutsey PL, Cushman M, Rosamond WD. Community prevalence of ideal cardiovascular health, by the American Heart Association definition, and relationship with cardiovascular disease incidence. J Am Coll Cardiol. 2011;57(16):1690-6.

11. Yang Q, Cogswell ME, Flanders WD, Hong Y, Zhang Z, Loustalot $\mathrm{F}$, et al. Trends in cardiovascular health metrics and associations with all-cause and CVD mortality among US adults. JAMA. 2012;307(12):1273-83. This analysis of National Health and Nutrition Examination Surveys mortality-linked data demonstrates the low prevalence of ideal cardiovascular health in the nation and the graded relationship between ideal levels of health behaviors and health factors and lower risk for all-cause and cardiovacular mortality.

12. Ford ES, Greenlund KJ, Hong Y. Ideal cardiovascular health and mortality from all causes and diseases of the circulatory system among adults in the United States. Circulation. 2012;125(8):98795. 
13. Rasmussen-Torvik LJ, Shay CM, Abramson JG, Friedrich CA, Nettleton JA, Prizment AE, et al. Ideal cardiovascular health is inversely associated with incident cancer: the Atherosclerosis Risk In Communities Study. Circulation. 2013;127(12):1270-5.

14. Reis JP, Loria CM, Launer LJ, Sidney S, Liu K, Jacobs Jr DR, et al. Cardiovascular health through young adulthood and cognitive functioning in midlife. Ann Neurol. 2013;73(2):170-9.

15. Espana-Romero V, Artero EG, Lee DC, Sui X, Baruth M, Ruiz JR, et al. A prospective study of ideal cardiovascular health and depressive symptoms. Psychosomatics. 2013;54(6):525-35.

16. Allen NB, Ning H, Huffman MD, Liu K, Reis J, Lloyd-Jones D. Maintenance of ideal cardiovascular health over 20 years and its impact on health related quality of life: the Coronary Artery Development in Young Adults (CARDIA) study. Circulation. 2013;128(22S):A15499 [abstract].

17. Bambs C, Kip KE, Dinga A, Mulukutla SR, Aiyer AN, Reis SE. Low prevalence of "ideal cardiovascular health" in a communitybased population: the heart strategies concentrating on risk evaluation (Heart SCORE) study. Circulation. 2011;123(8):850-7.

18. Dong C, Rundek T, Wright CB, Anwar Z, Elkind MS, Sacco RL. Ideal cardiovascular health predicts lower risks of myocardial infarction, stroke, and vascular death across whites, blacks, and hispanics: the northern Manhattan study. Circulation. 2012;125(24):2975-84.

19. Shay CM, Ning H, Allen NB, Carnethon MR, Chiuve SE, Greenlund $\mathrm{KJ}$, et al. Status of cardiovascular health in US adults: prevalence estimates from the National Health and Nutrition Examination Surveys (NHANES) 2003-2008. Circulation. 2012;125(1):45-56.

20. Go AS, Mozaffarian D, Roger VL, Benjamin EJ, Berry JD, Blaha MJ, et al. Heart disease and stroke statistics: 2014 update: a report from the American Heart Association. Circulation. 2014;129(3): e28-e292.

21. Fang J, Yang Q, Hong Y, Loustalot F. Status of cardiovascular health among adult Americans in the 50 states and the District of Columbia, 2009. J Am Heart Assoc. 2012;1(6):e005371.

22. Enos WF, Holmes RH, Beyer J. Coronary disease among United States soldiers killed in action in Korea; preliminary report. J Am Med Assoc. 1953;152(12):1090-3.

23. Berenson GS, Srinivasan SR, Bao W, Newman 3rd WP, Tracy RE, Wattigney WA. Association between multiple cardiovascular risk factors and atherosclerosis in children and young adults: the Bogalusa Heart Study. N Engl J Med. 1998;338(23):1650-6.

24. McGill Jr HC, McMahan CA. Determinants of atherosclerosis in the young. Pathobiological Determinants of Atherosclerosis in Youth (PDAY) Research Group. Am J Cardiol. 1998;82(10B): 30T-6T.

25. Juonala M, Magnussen CG, Venn A, Dwyer T, Burns TL, Davis PH, et al. Influence of age on associations between childhood risk factors and carotid intima-media thickness in adulthood: the Cardiovascular Risk in Young Finns Study, the Childhood Determinants of Adult Health Study, the Bogalusa Heart Study, and the Muscatine Study for the International Childhood Cardiovascular Cohort (i3C) Consortium. Circulation. 2010;122(24):2514-20.

26. Loria CM, Liu K, Lewis CE, Hulley SB, Sidney S, Schreiner PJ, et al. Early adult risk factor levels and subsequent coronary artery calcification: the CARDIA study. J Am Coll Cardiol. 2007;49(20): 2013-20.

27.• Laitinen TT, Pahkala K, Magnussen CG, Viikari JS, Oikonen M, Taittonen $\mathrm{L}$, et al. Ideal cardiovascular health in childhood and cardiometabolic outcomes in adulthood: the Cardiovascular Risk in Young Finns Study. Circulation. 2012;125(16):1971-8. Twenyone-year follow-up of participants in the Young Finns Study demonstrating that increased numbers of cardiovascular health behaviors and factors in childhood are associated with lower risk factors in adulthood.

28. van den Oord SC, Sijbrands EJ, ten Kate GL, van Klaveren D, van Domburg RT, van der Steen AF, et al. Carotid intima-media thickness for cardiovascular risk assessment: systematic review and meta-analysis. Atherosclerosis. 2013;228(1):1-11.

29.• Liu K, Daviglus ML, Loria CM, Colangelo LA, Spring B, Moller AC, et al. Healthy lifestyle through young adulthood and the presence of low cardiovascular disease risk profile in middle age: the Coronary Artery Risk Development in (Young) Adults (CARDIA) study. Circulation. 2012;125(8):996-1004. Demonstrates a graded relationship between healthy lifestyle factors in young adulthood with lower cardiovascular disease risk in middle age in the Coronary Artery Disease in Young Adults (CARDIA) cohort. This study helps affirm the importance of lifestyle factors in maintaining cardiovascular health.

30. Kulshreshtha A, Goyal A, Veledar E, McClellan W, Judd S, Eufinger SC, et al. Association between ideal cardiovascular health and carotid intima-media thickness: a twin study. J Am Heart Assoc. 2014;3(1):e000282.

31. Allen NB, Hwang S, Cupples LA, Levy D, Fox C, O'Donnell C, et al. The heritability of ideal cardiovascular health: the Framingham Heart Study. Circulation. 2010;122(21S):A17245.

32. Artinian NT, Fletcher GF, Mozaffarian D, Kris-Etherton P, Van Horn L, Lichtenstein AH, et al. Interventions to promote physical activity and dietary lifestyle changes for cardiovascular risk factor reduction in adults: a scientific statement from the American Heart Association. Circulation. 2010;122(4):406-41.

33. Burke LE, Styn MA, Sereika SM, Conroy MB, Ye L, Glanz K, et al Using mHealth technology to enhance self-monitoring for weight loss: a randomized trial. Am J Prev Med. 2012;43(1):20-6.

34. Spring B, Schneider K, McFadden HG, Vaughn J, Kozak AT, Smith $\mathrm{M}$, et al. Multiple behavior changes in diet and activity: a randomized controlled trial using mobile technology. Arch Intern Med. 2012;172(10):789-96. Clinical trial that demonstrates that a mobile health intervention targeting increased fruit and vegetable consumption and reduced sedentary activity produces more of an effect than traditional dietary and activity interventions. This study demonstrates the potential of mobile technologies to deliver multiple risk factor interventions more intensively and at a lower cost.

35. Pearson TA, Palaniappan LP, Artinian NT, Carnethon MR, Criqui MH, Daniels SR, et al. American Heart Association guide for improving cardiovascular health at the community level, 2013 update: a scientific statement for public health practitioners, healthcare providers, and health policy makers. Circulation. 2013;127(16):1730-53.

36. Mozaffarian D, Afshin A, Benowitz NL, Bittner V, Daniels SR, Franch HA, et al. Population approaches to improve diet, physical activity, and smoking habits: a scientific statement from the American Heart Association. Circulation. 2012;126(12):1514-63.

37. Koh HK, Sebelius KG. Promoting prevention through the Affordable Care Act. N Engl J Med. 2010;363(14):1296-9.

38. Frieden TR, Berwick DM. The "Million Hearts" initiative: preventing heart attacks and strokes. N Engl J Med. 2011;365(13):e27. 